\title{
ГРАНИЧНА ПОВЕДІНКА РОЗПОДІЛУ МОМЕНТУ РУЙНАЦІЇ МОДИФІКОВАНОГО ПРОЦЕСУ РИЗИКУ
}

For modified risk process with instantaneous reflection at the point $B>0$ under which the considered process

$$
\zeta(t)=\zeta_{B, u}(t), \quad \zeta(0)=u, 0 \leq u<B,
$$

returns in the initial state $u$, we investigate the limit behavior of generating function of the first ruin moment as $u \rightarrow B$ and $B \rightarrow \infty$.

Для модифікованого процесу ризику з миттевим відбиттям в точці $B>0$, при якому розглядуваний процес

$$
\zeta(t)=\zeta_{B, u}(t), \quad \zeta(0)=u, 0 \leq u<B,
$$

повертається в початковий стан $u$, досліджено граничну поведінку генератриси моменту першої руйнації при $u \rightarrow B$ та $B \rightarrow \infty$.

Нехай $U(t)=u+\xi(t)-$ класичний процес ризику, де $\xi(t)=c t-\mathrm{X}(t)-$ складний пуассонівський процес з додатним знесенням $c>0$ та від'ємними стрибками $\left\{\xi_{k}\right\}_{k=1}^{\infty}$. Интервали між сусідніми стрибками $\eta_{k}>0-$ показниково розподілені з параметром $\lambda>0$ і характеристична функція (х. ф.) процесу $\xi(t)$ має вигляд

$$
\begin{gathered}
\mathrm{E} e^{i \alpha \xi(t)}=\exp \{t \psi(\alpha)\}, \\
\psi(\alpha)=i \alpha c+\lambda \int_{-\infty}^{0}\left(e^{i \alpha x}-1\right) d F(x) .
\end{gathered}
$$

Введемо позначення основних функціоналів для початкового процесу $\xi(t)$ :

$$
\begin{gathered}
m=\mathrm{E} \xi(1)=c+\lambda \mathrm{E} \xi_{k}, \quad c>0, \mathrm{E} \xi_{k}<0, \\
\xi^{ \pm}(t)=\sup _{0 \leq s \leq t}(\inf ) \xi(s), \\
\tau^{ \pm}( \pm z)=\inf \{t>0: \pm \xi(t)>z\} \quad z>0, \\
\tau_{B}(u)=\inf \{t>0: u+\xi(t) \bar{\epsilon} t(0, B)\},
\end{gathered}
$$

які визначають відповідно екстремуми процесу, моменти першого виходу за рівень $\pm z$ та виходу з інтервалу $(0, B)$.

Розглядуваний в [1] модифікований процес ризику (м. п. р.) 3 миттєвим відбиттям у початковий стан $u, 0<u<B, v=B-u$, визначається стохастичним співвідношенням

$$
\zeta(t)=\zeta_{B, u}(t)=\left\{\begin{array}{c}
u+\xi(t), t<\tau^{+}(v)=T_{1}, v=B-u ; \\
\zeta\left(t-T_{1}\right), t>T_{1} .
\end{array}\right.
$$

Класичний процес ризику $U(t)$ можна виразити сумою

$$
U(t)=\zeta(t)+X(t)
$$

де процес $X(t)$, що називається дивідендним, визначається співвідношенням 


$$
X(t)=X_{B, u}(t)=n(t) v, \quad v=B-u,
$$

$n(t)$-кількість досягнень процесом $\zeta(\cdot)$ рівня $B$ на інтервалі $[0 ; t]$.

Для вивчення граничної поведінки розподілу моменту першої руйнації процесу $\zeta(t)$

$$
T_{u}^{B}=\inf \left\{t>0: \zeta_{B, u}(t)<0\right\}
$$

введемо допоміжні позначення і сформулюємо деякі допоміжні твердження 3 $[2,3]$. Нехай $\theta_{s}-$ показниково розподілена випадкова величина (незалежна від $\xi(t))$ з параметром $s>0$. Тоді позначимо

$$
\begin{gathered}
P(s, x)=\mathrm{P}\left\{\xi\left(\theta_{s}\right)<x\right\}, \quad-\infty<x<\infty, \\
P_{ \pm}(s, \pm x)=\mathrm{P}\left\{\xi^{ \pm}\left(\theta_{s}\right)< \pm x\right\}, \quad x \geq 0, \\
\varphi_{ \pm}(s, \alpha)=\mathrm{E} e^{i \alpha \xi^{ \pm}\left(\theta_{s}\right)} .
\end{gathered}
$$

Справедлива така лема (див. [2]).

Лема. Для неперервного зверху процесу $\xi(t)$

$$
\varphi_{+}(s, \alpha)=\frac{\rho(s)}{\rho(s)+i \alpha},
$$

де $\rho(s)-\epsilon$ дний додатний корінь рівняння $\psi(i \rho)=s, s>0$,

$$
\begin{gathered}
\varphi_{-}(s, \alpha)=p_{-}(s)+\frac{1}{\rho(s)} \int_{-\infty}^{0} e^{i \alpha x} d_{x} P^{\prime}(s, x)+\mathrm{E}\left[e^{i \alpha \xi\left(\theta_{s}\right)}, \xi\left(\theta_{s}\right)<0\right], \\
P^{\prime}(s, x)=\frac{\partial}{\partial(x)} P(s, x), \quad x \neq 0, \quad p_{-}(s)=\mathrm{P}\left\{\xi^{-}\left(\theta_{s}\right)=0\right\} .
\end{gathered}
$$

При умові $m>0$ існує $\lim _{s \rightarrow 0} \rho(s) s^{-1}=\rho^{\prime}(0)=\frac{1}{m}$ і абсолютний мінімум

$$
\xi^{-}=\inf _{t<\infty} \xi(t),
$$

має невироджений розподіл $(x<0)$

$$
\mathrm{P}\left\{\xi^{-}<x\right\}=\frac{1}{\rho^{\prime}(0)} \frac{d}{d x} \int_{0}^{\infty} \mathrm{P}\{\xi(t)<x\} d t .
$$

На основі розглянутих позначень і співвідношень леми введемо позначення резольвентної функції і потенціалу (див. $[2,3]$ )

$$
\begin{gathered}
R_{s}(x)=s^{-1} \rho(s) \int_{-0}^{x} e^{\rho(s)(x-y)} d \mathrm{P}\left\{-\xi^{-}\left(\theta_{s}\right)<y\right\}, \\
R(x)=\lim _{s \rightarrow 0} R_{s}(x), \quad x>0,
\end{gathered}
$$

а також м. п. р. із затримкою в $B$

та моменту пї руйнації

$$
\eta(t)=\eta_{B, u}(t)=\left\{\begin{array}{c}
u+\xi(t), \quad t<T_{1}=\tau^{+}(v) ; \\
B, \quad T_{1}<t<T_{1}+\eta_{1}=T_{*} ; \\
\eta_{B, u}\left(t-T_{*}\right), \quad t>T_{*},
\end{array}\right.
$$

$$
T_{B}(u)=\inf \left\{t>0: \eta_{B, u}(t)<0\right\} .
$$


Із стохастичного співвідношення

випливає (див. [3])

$$
T_{u}^{B}=\left\{\begin{array}{c}
\tau^{-}(-u), \quad \tau^{-}(-u)<\tau^{+}(v) \\
\tau^{+}(v)+T_{u}^{B}, \quad \tau^{+}(v) \leq \tau^{-}(-u)
\end{array}\right.
$$

$$
\mathrm{E} e^{-s T_{u}^{B}}=\frac{R_{s}(B) E e^{-s \tau_{B}(u)}-R_{s}(u)}{R_{s}(B)-R_{s}(u)},
$$

а для $\tau_{B}(u)$ та $T_{B}(u)$, згідно з [2,3], справедливі співвідношення

$$
\begin{aligned}
& \mathrm{E} e^{-s \tau_{B}(u)}=1-s\left(\frac{R_{s}(u)}{R_{s}(B)} \int_{0}^{B} R_{s}(y) d y-\int_{0}^{u} R_{s}(y) d y\right), \\
& \mathrm{E} e^{-s T_{B}(u)}=1-s\left(\frac{R_{s}(B)}{R_{s}^{\prime}(B)} R_{s}(u) d y-\int_{0}^{u} R_{s}(y) d y\right), \\
& R_{s}^{\prime}(u)=\frac{d}{d u} R_{s}(u), \quad R_{s}^{\prime}(B)=\left.\frac{d}{d u} R_{s}(u)\right|_{u=B-0} .
\end{aligned}
$$

На основі (9) - (11) встановлюється таке твердження.

Теорема 1. При и $\rightarrow$ в розподіл $T_{u}^{B}$-моменту периої руйнації м. п. p. $\zeta(t)$ - збігається з розподілом $T_{B}(B)$ - ломентом периої руйнації процесу $\eta_{B, B}(t)$,

$$
\lim _{u \rightarrow B} \mathrm{E} e^{-s T_{u}^{B}}=\mathrm{E} e^{-s T_{B}(B)}
$$

Доведення базується на граничному переході $(u \rightarrow B)$ в (9) з використанням правила Лопіталя і врахуванням того, що

$$
\left.\frac{d}{d u}\left(\mathrm{E} e^{-s \tau_{B}(u)}\right)\right|_{u=B}=s\left(R_{s}(B)-\frac{R_{s}^{\prime}(B)}{R_{s}(B)} \int_{0}^{B} R_{s}(y) d y\right) .
$$

В результаті цього з (9) випливає

$$
\begin{gathered}
\lim _{u \rightarrow B} \mathrm{E} e^{-s T_{u}^{B}}=\lim _{u \rightarrow B} \frac{R_{s}(B) \frac{d}{d u}\left(E e^{-s \tau_{B}(u)}\right)-R_{s}^{\prime}(u)}{-R_{s}^{\prime}(u)}= \\
=1+s\left(\int_{0}^{B} R_{s}(y) d y-R_{s}^{2}(B)\left[R_{s}^{\prime}(B)\right]^{-1}\right),
\end{gathered}
$$

звідки на основі (11) при $u \rightarrow B$ встановлюється потрібне співвідношення (12).

Гранична поведінка $T_{u}^{B}$ при $B \rightarrow 0$ залежить від знаку $m=\mathrm{E} \xi(1)$ і визначається на основі (9) при $m>0$ розподілом $\xi^{-}$, а при $m \leq 0$ поведінкою розподілу $\tau_{B}(u)$-моменту першого виходу $\xi(t)$ з інтервалу $(0, B)$.

Теорема 2. 1. Нехай $m>0$; тодi при $u>0$ іскінченних $s>0$

$$
\begin{gathered}
\lim _{B \rightarrow \infty} \mathrm{E} e^{-\frac{s}{B} T_{u}^{B}}=\frac{\mathrm{P}\left\{\xi^{-} \leq-u\right\}+\mathrm{P}\left\{\xi^{-}>-u\right\}\left(e^{-\frac{s}{m}}-1\right)}{\mathrm{P}\left\{\xi^{-} \leq-u\right\}}, \\
\lim _{B \rightarrow \infty} \mathrm{E} e^{-s B^{-1} T_{0}^{B}}=\frac{1}{m} \frac{\mathrm{P}\left\{\xi^{-}>-u\right\}}{\mathrm{P}\left\{\xi^{-} \leq-u\right\}} .
\end{gathered}
$$


При $и=0$ і скінченних $s>0$

$$
\begin{gathered}
\lim _{B \rightarrow \infty} \mathrm{E} e^{-s B^{-1} T_{0}^{B}}=1+\mathrm{P}\left\{\xi^{-}=0\right\}\left(e^{-\frac{s}{m}}-1\right), \\
\lim _{B \rightarrow \infty} \mathrm{E} T_{0}^{B} B^{-1}=\frac{1}{m} \mathrm{P}\left\{\xi^{-}=0\right\} .
\end{gathered}
$$

2. Нехай $m=0, D \xi(1)=\sigma^{2}<\infty ;$ тоді при $u \approx \beta B, 0<\beta<1$, і скінченних $s>0$,

$$
\lim _{B \rightarrow \infty} \mathrm{E} e^{-s B^{-2} T_{u}^{B}}=\frac{\operatorname{sh}(1-\beta) \sqrt{2 s} \sigma^{-1}}{\operatorname{sh} \sqrt{2 s} \sigma^{-1}-\operatorname{sh} \beta \sqrt{2 s} \sigma^{-1}} .
$$

3. Нехай $m<0$, тодi при $u \geq 0$

$$
\lim _{B \rightarrow \infty} \mathrm{E} e^{-s T_{u}^{B}}=\lim _{B \rightarrow \infty} \mathrm{E} e^{-s \tau_{B}(u)}=\mathrm{E} e^{-s \tau^{-}(-u)} .
$$

Доведення. 1. При $m>0$ з теореми 5.2. 1 (див. [3, с. 156]) випливає

$$
\lim _{B \rightarrow \infty} \mathrm{E} e^{-s B^{-1} \tau_{B}(u)}=1-m R(u)\left(1-e^{-\frac{s}{m}}\right),
$$

де

$$
R(u)=\rho^{\prime}(0) \mathrm{P}\left\{-\xi^{-} \leq u\right\} \quad \rho^{\prime}(0)=m^{-1} .
$$

Тоді з (9) при скінченних $s$ одержуємо

$$
\begin{gathered}
\lim _{B \rightarrow \infty} R_{s B^{-1}}(u)=R(u), \quad u>0, \\
\lim _{B \rightarrow \infty} \mathrm{E} e^{-s B^{-1} T_{u}^{B}}=\lim _{B \rightarrow \infty} \frac{R_{s B^{-1}}(B) \mathrm{E} e^{-s B^{-1} \tau_{B}(u)}-R_{s B^{-1}}(u)}{R_{s B^{-1}}(B)-R_{s B^{-1}}(u)}= \\
=\frac{1-\mathrm{P}\left\{-\xi^{-}<u\right\}+\left(e^{-\frac{s}{m}}-1\right) \mathrm{P}\left\{-\xi^{-}<u\right\}}{\mathrm{P}\left\{-\xi^{-} \geq u\right\}},
\end{gathered}
$$

i, таким чином, перше співвідношення в (13) доведено. 3 нього виводиться й друге співвідношення в (13) граничним переходом $(s \rightarrow 0)$. При $m=0$ і скінченних $s>0$ із (13) випливають обидва співвідношення (14).

2. При $m=0$ та $\sigma^{2}<\infty$ з теореми 5.2. 2 (див. [3, с. 156]) при $u \approx \beta B$, $0<\beta<1$,

$$
\lim _{B \rightarrow \infty} \mathrm{E} e^{-s B^{-2} \tau_{B}(u)}=\frac{\operatorname{sh}(1-\beta) \sqrt{2 s} \sigma^{-1}+\operatorname{sh} \beta \sqrt{2 s} \sigma^{-1}}{\operatorname{sh} \sqrt{2 s} \sigma^{-1}} .
$$

При цих же умовах, згідно з теоремою 7.6 (див. [3, с. 131]) для скінченних $s>0$

$$
\lim _{B \rightarrow \infty} \frac{R_{s B^{-2}}(\beta B)}{R_{s B^{-2}}(B)}=\frac{\operatorname{sh} \beta \sqrt{2 s} \sigma^{-1}}{\operatorname{sh} \sqrt{2 s} \sigma^{-1}}
$$




$$
\begin{gathered}
\lim _{B \rightarrow \infty} \mathrm{E} e^{-s B^{-2} T_{u}^{B}}=\lim _{B \rightarrow \infty}\left[\mathrm{E} e^{-s B^{-2} \tau_{B}(u)}-\frac{R_{s B^{-2}}(\beta B)}{R_{s B^{-2}}(B)}\right] \times \\
\times\left(1-\frac{R_{s B^{-2}}(\beta B)}{R_{s B^{-2}}(B)}\right)^{-1}=\left[\frac{\operatorname{sh}(1-\beta) \sqrt{2 s} \sigma^{-1}+\operatorname{sh} \beta \sqrt{2 s} \sigma^{-1}}{\operatorname{sh} \sqrt{2 s} \sigma^{-1}}-\frac{\operatorname{sh} \beta \sqrt{2 s} \sigma^{-1}}{\operatorname{sh} \sqrt{2 s} \sigma^{-1}}\right] \times \\
\times\left(1-\frac{\operatorname{sh} \beta \sqrt{2 s} \sigma^{-1}}{\operatorname{sh} \sqrt{2 s} \sigma^{-1}}\right)^{-1}=\frac{\operatorname{sh}(1-\beta) \sqrt{2 s} \sigma^{-1}}{\operatorname{sh} \sqrt{2 s} \sigma^{-1}-\operatorname{sh} \beta \sqrt{2 s} \sigma^{-1}} .
\end{gathered}
$$

Таким чином, співвідношення (15) доведено.

3. Нехай $m<0$. Зауважимо, що з точки зору теорії страхування цей випадок не $є$ цікавим. Ми розглянемо його для повноти викладу і відмітимо, що в цьому випадку, згідно з результатами $[2,3]$,

$$
\begin{gathered}
\lim _{B \rightarrow \infty} Q^{B}(s, u)=\lim _{B \rightarrow \infty} \mathrm{E}\left[e^{-s \tau_{B}(u)}, \tau^{+}(u)<\tau^{-}(-u)\right]=0, \\
\lim _{B \rightarrow \infty} \mathrm{E} e^{-s \tau_{B}(u)}=\mathrm{E} e^{-s \tau^{-}(-u)}, \quad u \geq 0 .
\end{gathered}
$$

На основі співвідношення

$$
\mathrm{E} e^{-s T_{u}^{B}}=\left(\mathrm{E} e^{-s \tau_{B}(u)}-Q^{B}(s, u)\right)\left(1-Q^{B}(s, u)\right)^{-1}
$$

та останніх двох граничних співвідношень встановлюється справедливість формули (16). Теорему 2 доведено.

Нехай $S_{v}^{+}(0)=0$, а при $n>0$

$$
S_{v}^{+}(n)=\sum_{k=0}^{n} \tau_{k}^{+}(v), \quad \tau_{k}^{+}(v)=\tau^{+}(v) .
$$

Позначимо функцію відновлення для $S_{v}^{+}(n)$ через

$$
H_{v}(t)=\sum_{n=0}^{\infty} \mathrm{P}\left\{S_{v}^{+}(n)<t\right\}
$$

і сформулюємо наступне твердження.

Теорема 3. При $m>0$ середнє значення $X_{B, u}(t)$ визначається співвідноченнял

$$
\mathrm{E} X_{B, u}(t)=v\left(H_{v}(t)-1\right), \quad \mathrm{E} X_{B, u}(t) \approx t m, t \rightarrow \infty .
$$

Доведення. Оскільки число відновлень $n\left(\theta_{s}\right)$ має геометричний розподіл

$$
\begin{gathered}
\mathrm{P}\left\{n\left(\theta_{s}\right)=k\right\}=\left(1-q_{v}(s)\right) q_{v}^{k}(s), \quad k \geq 0, \\
q_{v}(s)=e^{-v p(s)}=p\left\{\xi^{+}\left(\theta_{s}\right)>v\right\}, \quad s>0, v=B-u>0,
\end{gathered}
$$

To

$$
\begin{gathered}
\mathrm{E} X_{B, u}\left(\theta_{s}\right)=v e^{-\rho(s) v}\left(1-e^{-v \rho(s)}\right)^{-1}= \\
=v \mathrm{P}\left\{\xi^{+}\left(\theta_{s}\right)>v\right\} \sum_{k=0}^{\infty}\left(\mathrm{E} e^{-s \tau^{+}(v)}\right)^{k}= \\
=v\left(\sum_{n=0}^{\infty} \mathrm{E} e^{-s S_{v}^{+}(n)}-1\right) .
\end{gathered}
$$


Отже, після обернення відносно $s$ матимемо перше співвідношення в (17).

Згідно з теоремою відновлення при $t \rightarrow \infty$

$$
H_{v}(t) \approx \frac{t}{\mathrm{E} \tau^{+}(v)}=\frac{t}{v \rho^{\prime}(0)} .
$$

Тому з (17) та (18) випливає, що при $t \rightarrow \infty$

$$
\mathrm{E} X_{B, u}(t) \sim \frac{t}{\rho^{\prime}(0)}=m t .
$$

Згідно з результатами [4, с. 318$]$

$$
H_{v}(t)=\frac{t}{\mathrm{E} \tau^{+}(v)}+\frac{\mathrm{E} \gamma_{v}^{+}(t)}{\mathrm{E} \tau^{+}(v)},
$$

де

$$
\gamma_{v}^{+}(t)=S_{v}^{+}\left(\eta_{t}^{+}\right)-t, \quad \eta_{t}^{+}=\min \left\{n: S_{v}^{+}(n) \geq t\right\}
$$

3 теореми 4.9 (див. [5, с. 292]) випливає

$$
\mathrm{E} e^{-v \gamma_{v}^{+}\left(\theta_{\mu}\right)} u^{\tau_{v}^{+}\left(\theta_{\mu}\right)}=\frac{\mu u}{\mu-v} \frac{\mathrm{E} e^{-v \tau^{+}(v)}-\mathrm{E} e^{-\mu \tau^{+}(v)}}{1-u \mathrm{E} e^{-\mu \tau^{+}(v)}} .
$$

Отже, твірна функція $\gamma_{v}^{+}\left(\theta_{\mu}\right)$ має вигляд

$$
\begin{gathered}
g(v ; \mu, v)=\mathrm{E} e^{-v \gamma_{v}^{+}\left(\theta_{\mu}\right)}=\frac{\mu u}{\mu-v} \frac{\mathrm{E} e^{-v \tau^{+}(v)}-\mathrm{E} e^{-\mu \tau^{+}(v)}}{1-\mathrm{E} e^{-\mu \tau^{+}(v)}}= \\
=\frac{\mu}{\mu-v} \frac{e^{-v \rho(v)}-e^{-v \rho(\mu)}}{1-e^{-v \rho(\mu)}} .
\end{gathered}
$$

При $\mathrm{E} \xi(1)>0, \rho(\mu) \rightarrow 0$ при $\mu \rightarrow 0, \frac{\rho(\mu)}{\mu} \underset{\mu \rightarrow 0}{\rightarrow} \rho^{\prime}(0)>0$,

$$
\mathrm{E} e^{-v \gamma_{v}^{+}(\infty)}=\frac{1-e^{-v \rho(v)}}{v v \rho^{\prime}(0)}
$$

Знайдемо $\mathrm{E} \gamma_{v}^{+}\left(\theta_{\mu}\right)$, скориставшись похідною $g_{v}^{\prime}=\frac{\partial g}{\partial v}$,

$$
\begin{gathered}
g_{v}^{\prime}(v ; \mu, v)=\frac{\mu}{(\mu-v)^{2}} \frac{e^{-v \rho(v)}-e^{-v \rho(\mu)}}{1-e^{-v \rho(\mu)}}- \\
-\frac{\mu}{\mu-v} v \rho^{\prime}(v) \frac{e^{-v \rho(v)}}{1-e^{-v \rho(\mu)}}, \\
\mathrm{E} \gamma_{v}^{+}\left(\theta_{\mu}\right)=-g_{v}^{\prime}(0 ; \mu, v)=\frac{v \rho^{\prime}(0)}{1-e^{-v \rho(\mu)}}-\frac{1}{\mu}, \\
\mathrm{E} \gamma_{v}^{+}\left(\theta_{\mu}\right)=\frac{v \rho^{\prime}(0)}{\mathrm{P}\left\{\xi^{+}\left(\theta_{\mu}\right) \leq v\right\}}-\frac{1}{\mu} .
\end{gathered}
$$




$$
\mathrm{E} \gamma_{v}^{+}(t)=\frac{\mathrm{E} \tau^{+}(v)}{\mathrm{P}\left\{\xi^{+}\left(\theta_{\mu}\right) \leq v\right\}}-t
$$

отже, при $m>0$

$$
H_{v}(t)=\frac{1}{P\left\{\xi^{+}(t) \leq v\right\}},
$$

і для скінченних $v>0$ з (18) знаходимо

$$
\mathrm{P}\left\{\xi^{+}(t) \leq v\right\} \approx \frac{v}{m t}, \quad t \rightarrow \infty .
$$

1. Гусак Д. В. Про одну модель осцилюючого випадкового блукання, що описує процес ризику //Допов. НАН Украйни. - 1998. - №4. - С. 7 - 11.

2. Братийчук Н. С., Гусак Д. В. Граничные задачи для процессов с независимыми приращениями. - Киев: Наук. думка, 1990. -264 с.

3. Королюк В. С., Братийчук Н. С., Пирджапов Б. Граничные задачи для случайных блужданий. - Ашхабад: Ылым, 1987 . -256 с.

4. Боровков А. А. Вероятностные процессы в теории массового обслуживания. - М.: Наука, 1972. $-368 \mathrm{c}$.

5. Гусак Д. В. Граничні задачі для процесів з незалежними приростами на скінченних ланцюгах Маркова та для папівмарковських процесів. - Київ: Ін-т математики НАН України, 1998. $-320 \mathrm{c}$. 